\title{
Discrete approximation to solution flows of Tanaka's SDE related to Walsh Brownian motion
}

Hatem Hajri

\begin{abstract}
In a previous work, we have defined a Tanaka's SDE related to Walsh Brownian motion which depends on kernels. It was shown that there are only one Wiener solution and only one flow of mappings solving this equation. In the terminology of Le Jan and Raimond, these are respectively the stronger and the weaker among all solutions. In this paper, we obtain these solutions as limits of discrete models.
\end{abstract}

\section{Introduction and main results}

Consider Tanaka's equation:

$$
\varphi_{s, t}(x)=x+\int_{s}^{t} \operatorname{sgn}\left(\varphi_{s, u}(x)\right) d W_{u}, \quad s \leq t, x \in \mathbb{R},
$$

where $\operatorname{sgn}(x)=1_{\{x>0\}}-1_{\{x \leq 0\}}, W_{t}=W_{0, t} 1_{\{t>0\}}-W_{t, 0} 1_{\{t \leq 0\}}$ and $\left(W_{s, t}, s \leq t\right)$ is a real white noise on a probability space $(\Omega, \mathscr{A}, \mathbb{P})$ (see Definition 1.10 [6]). This is an example of a stochastic differential equation which admits a weak solution but has no strong solution. If $K$ is a stochastic flow of kernels (see Section 2.1 [5]) and $W$ is a real white noise, then by definition, $(K, W)$ is a solution of Tanaka's SDE if for all $s \leq t, x \in \mathbb{R}, f \in C_{b}^{2}(\mathbb{R})$ ( $f$ is $C^{2}$ on $\mathbb{R}$ and $f^{\prime}, f^{\prime \prime}$ are bounded),

$$
K_{s, t} f(x)=f(x)+\int_{s}^{t} K_{s, u}\left(f^{\prime} \operatorname{sgn}\right)(x) W(d u)+\frac{1}{2} \int_{s}^{t} K_{s, u} f^{\prime \prime}(x) d u \text { a.s. }
$$

When $K=\delta_{\varphi}$ is a flow of mappings, $K$ solves (2) if and only if $\varphi$ solves (1) by Itô's formula. In [7], Le Jan and Raimond have constructed the unique flow of mappings associated to (1). It was shown also that

Hatem Hajri

Université Paris Sud 11, e-mail: Hatem.Hajri@math.u-psud.fr 


$$
K_{s, t}^{W}(x)=\delta_{x+\operatorname{sgn}(x) W_{s, t}} 1_{\left\{t \leq \tau_{s, x}\right\}}+\frac{1}{2}\left(\delta_{W_{s, t}^{+}}+\delta_{-W_{s, t}^{+}}\right) 1_{\left\{t>\tau_{s, x}\right\}}, \quad s \leq t, x \in \mathbb{R},
$$

is the unique $\mathscr{F}^{W}$ adapted solution (Wiener flow) of (2) where

$$
\tau_{s, x}=\inf \left\{r \geq s: W_{s, r}=-|x|\right\}, W_{s, t}^{+}:=W_{s, t}-\inf _{u \in[s, t]} W_{s, u}
$$

In [5], an extension of (2) in the case of Walsh Brownian motion was defined as follows

Definition 1 Fix $N \in \mathbb{N}^{*}, \alpha_{1}, \cdots, \alpha_{N}>0$ such that $\sum_{i=1}^{N} \alpha_{i}=1$ and consider the graph $G$ consisting of $N$ half lines $\left(D_{i}\right)_{1 \leq i \leq N}$ emanating from 0 (see Figure 1 ).

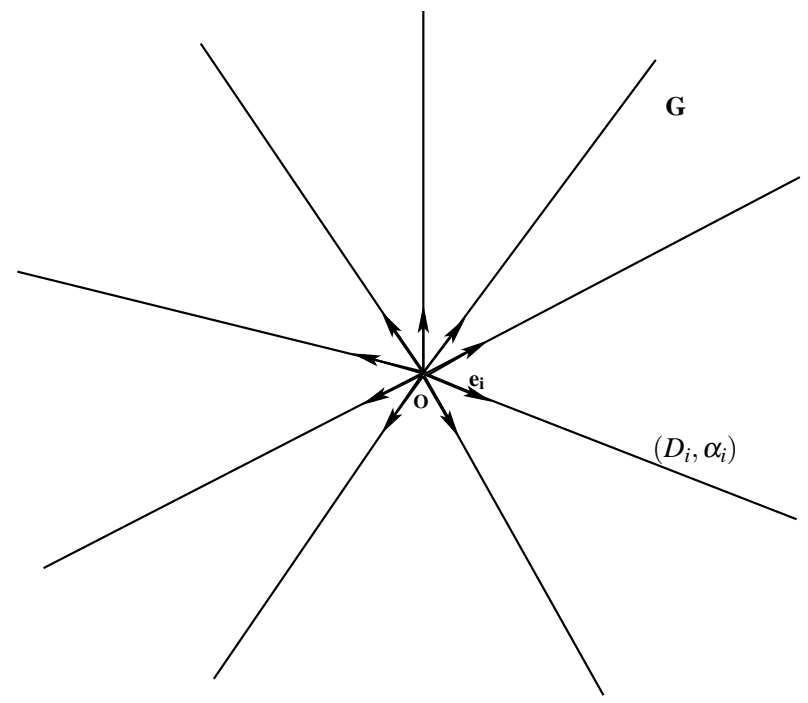

Fig. 1 Graph $G$.

Let $\mathbf{e}_{i}$ be a vector of modulus 1 such that $D_{i}=\left\{h \mathbf{e}_{i}, h \geqslant 0\right\}$ and define for all $z \in G, \mathbf{e}(z)=\mathbf{e}_{i}$ if $z \in D_{i}, z \neq 0$ (convention $\mathbf{e}(0)=\mathbf{e}_{N}$ ). Define the following distance on $G$ :

$$
d\left(h \mathbf{e}_{i}, h^{\prime} \mathbf{e}_{j}\right)= \begin{cases}h+h^{\prime} & \text { if } i \neq j,\left(h, h^{\prime}\right) \in \mathbb{R}_{+}^{2}, \\ \left|h-h^{\prime}\right| & \text { if } i=j,\left(h, h^{\prime}\right) \in \mathbb{R}_{+}^{2} .\end{cases}
$$

For $x \in G$, we will use the simplified notation $|x|:=d(x, 0)$.

We equip $G$ with its Borel $\sigma$-field $\mathscr{B}(G)$ and set $G^{*}=G \backslash\{0\}$. Let $C_{b}^{2}\left(G^{*}\right)$ be the space of all $f: G \longrightarrow \mathbb{R}$ such that $f$ is continuous on $G$ and has bounded first and second derivatives $\left(f^{\prime}\right.$ and $\left.f^{\prime \prime}\right)$ on $G^{*}$ (here $f^{\prime}(z)$ is the derivative of $f$ at $z$ in the 
direction $\mathbf{e}(z)$ for all $z \neq 0)$, both $\lim _{z \rightarrow 0, z \in D_{i}, z \neq 0} f^{\prime}(z)$ and $\lim _{z \rightarrow 0, z \in D_{i}, z \neq 0} f^{\prime \prime}(z)$ exist for all $i \in[1, N]$. Define

$$
D\left(\alpha_{1}, \cdots, \alpha_{N}\right)=\left\{f \in C_{b}^{2}\left(G^{*}\right): \sum_{i=1}^{N} \alpha_{i} \lim _{z \rightarrow 0, z \in D_{i}, z \neq 0} f^{\prime}(z)=0\right\} .
$$

Now, Tanaka's SDE on G extended to kernels is the following (see Remarks 3 (1) in [5] for a discussion of its origin).

Tanaka's equation on $G$. On a probability space $(\Omega, \mathscr{A}, \mathbb{P})$, let $W$ be a real white noise and $K$ be a stochastic flow of kernels on $G$. We say that $(K, W)$ solves $(T)$ if for all $s \leq t, f \in D\left(\alpha_{1}, \cdots, \alpha_{N}\right), x \in G$,

$$
K_{s, t} f(x)=f(x)+\int_{s}^{t} K_{s, u} f^{\prime}(x) W(d u)+\frac{1}{2} \int_{s}^{t} K_{s, u} f^{\prime \prime}(x) d u \text { a.s. }
$$

If $K=\delta_{\varphi}$ is a solution of $(T)$, we just say that $(\varphi, W)$ solves $(T)$.

Equation $(T)$ is a particular case of an equation $(E)$ studied in [5] (it corresponds to $\varepsilon=1$ with the notations of [5]). It was shown (see Corollary 2 [5]) that if $(K, W)$ solves $(T)$, then $\sigma(W) \subset \sigma(K)$ and therefore one can just say that $K$ solves $(T)$. We also recall

Theorem 1. [5] There exists a unique Wiener flow $K^{W}$ (resp. flow of mappings $\varphi$ ) which solves $(T)$.

As described in Theorem 1 [5], the unique Wiener solution of $(T)$ is simply

$$
K_{s, t}^{W}(x)=\delta_{x+\mathbf{e}(x) W_{s, t}} 1_{\left\{t \leq \tau_{s, x}\right\}}+\sum_{i=1}^{N} \alpha_{i} \delta_{\mathbf{e}_{i} W_{s, t}^{+}} 1_{\left\{t>\tau_{s, x}\right\}}
$$

where

$$
\tau_{s, x}=\inf \left\{r \geq s: x+\mathbf{e}(x) W_{s, r}=0\right\}=\inf \left\{r \geq s: W_{s, r}=-|x|\right\} .
$$

However, the construction of the unique flow of mappings $\varphi$ associated to $(T)$ relies on flipping Brownian excursions and is more complicated. Another construction of $\varphi$ using Kolmogorov extension theorem can be derived from Section 4.1 [7] similarly to Tanaka's equation. Here, we restrict our attention to discrete models.

The one point motion associated to any solution of $(T)$ is the Walsh Brownian motion $W\left(\alpha_{1}, \cdots, \alpha_{N}\right)$ on $G$ (see Proposition 3 [5]) which we define as a strong Markov process with càdlàg paths, state space $G$ and Feller semigroup $\left(P_{t}\right)_{t \geq 0}$ as given in Section 2.2 [5]. When $N=2$, it corresponds to the famous skew Brownian motion [4].

Our first result is the following Donsker approximation of $W\left(\alpha_{1}, \cdots, \alpha_{N}\right)$

Proposition 1 Let $M=\left(M_{n}\right)_{n \geq 0}$ be a Markov chain on $G$ started at 0 with stochastic matrix $Q$ given by: 


$$
Q\left(0, \mathbf{e}_{i}\right)=\alpha_{i}, \quad Q\left(n \mathbf{e}_{i},(n+1) \mathbf{e}_{i}\right)=Q\left(n \mathbf{e}_{i},(n-1) \mathbf{e}_{i}\right)=\frac{1}{2} \forall i \in[1, N], n \in \mathbb{N}^{*} .
$$

Let $t \longmapsto M(t)$ be the linear interpolation of $\left(M_{n}\right)_{n \geq 0}$ and $M_{t}^{n}=\frac{1}{\sqrt{n}} M(n t), n \geq 1$. Then

$$
\left(M_{t}^{n}\right)_{t \geq 0} \underset{n \rightarrow+\infty}{\stackrel{\text { law }}{\longrightarrow}}\left(Z_{t}\right)_{t \geq 0}
$$

in $C\left(\left[0,+\infty[, G)\right.\right.$ where $Z$ is an $W\left(\alpha_{1}, \cdots, \alpha_{N}\right)$ started at 0 .

This result extends that of [2] who treated the case $\alpha_{1}=\cdots=\alpha_{N}=\frac{1}{N}$ and of course the Donsker theorem for the skew Brownian motion (see [1] for example). We show in fact that Proposition 1 can be deduced immediately from the case $N=2$.

In this paper we study the approximation of flows associated to $(T)$. Among recent papers on the approximation of flows, let us mention [8] where the author construct an approximation for the Harris flow and the Arratia flow.

Let $G_{\mathbb{N}}=\{x \in G ;|x| \in \mathbb{N}\}$ and $\mathscr{P}(G)$ (resp. $\mathscr{P}\left(G_{\mathbb{N}}\right)$ ) be the space of all probability measures on $G$ (resp. $\left.G_{\mathbb{N}}\right)$. We now come to the discrete description of $\left(\varphi, K^{W}\right)$ and introduce

Definition 2 (Discrete flows) We say that a process $\psi_{p, q}(x)$ (resp. $\left.N_{p, q}(x)\right)$ indexed by $\left\{p \leq q \in \mathbb{Z}, x \in G_{\mathbb{N}}\right\}$ with values in $G_{\mathbb{N}}$ (resp. $\mathscr{P}\left(G_{\mathbb{N}}\right)$ ) is a discrete flow of mappings (resp. kernels) on $G_{\mathbb{N}}$ if:

(i) The family $\left\{\psi_{i, i+1} ; i \in \mathbb{Z}\right\}$ (resp. $\left\{N_{i, i+1} ; i \in \mathbb{Z}\right\}$ ) is independent.

(ii) $\forall p \in \mathbb{Z}, x \in G_{\mathbb{N}}, \psi_{p, p+2}(x)=\psi_{p+1, p+2}\left(\psi_{p, p+1}(x)\right)$

(resp. $\left.N_{p, p+2}(x)=N_{p, p+1} N_{p+1, p+2}(x)\right)$ a.s. where

$$
N_{p, p+1} N_{p+1, p+2}(x, A):=\sum_{y \in G_{\mathbb{N}}} N_{p+1, p+2}(y, A) N_{p, p+1}(x,\{y\}) \text { for all } A \subset G_{\mathbb{N}} .
$$

We call (ii), the cocycle or flow property.

The main difficulty in the construction of the flow $\varphi$ associated to (1) [7] is that it has to keep the consistency of the flow. This problem does not arise in discrete time. Starting from the following two remarks,

(i) $\varphi_{s, t}(x)=x+\operatorname{sgn}(x) W_{s, t}$ if $s \leq t \leq \tau_{s, x}$,

(ii) $\left|\varphi_{s, t}(0)\right|=W_{s, t}^{+}$and $\operatorname{sgn}\left(\varphi_{s, t}(0)\right)$ is independent of $W$ for all $s \leq t$,

one can easily expect the discrete analogous of $\varphi$ as follows: consider an original random walk $S$ and a family of signs $\left(\eta_{i}\right)$ which are independent. Then

(i) a particle at time $k$ and position $n \neq 0$, just follows what the $S_{k+1}-S_{k}$ tells him (goes to $n+1$ if $S_{k+1}-S_{k}=1$ and to $n-1$ if $S_{k+1}-S_{k}=-1$ ),

(ii)a particle at 0 at time $k$ does not move if $S_{k+1}-S_{k}=-1$, and moves according to $\eta_{k}$ if $S_{k+1}-S_{k}=1$.

The situation on a finite half-lines is very close. Let $S=\left(S_{n}\right)_{n \in \mathbb{Z}}$ be a simple random walk on $\mathbb{Z}$, that is $\left(S_{n}\right)_{n \in \mathbb{N}}$ and $\left(S_{-n}\right)_{n \in \mathbb{N}}$ are two independent simple random walks on $\mathbb{Z}$ and $\left(\eta_{i}\right)_{i \in \mathbb{Z}}$ be a sequence of i.i.d random variables with law $\sum_{i=1}^{N} \alpha_{i} \delta_{\mathbf{e}_{i}}$ which is 
independent of $S$. For $p \leq n$, set

$$
S_{p, n}=S_{n}-S_{p}, S_{p, n}^{+}=S_{n}-\min _{h \in[p, n]} S_{h}=S_{p, n}-\min _{h \in[p, n]} S_{p, h}
$$

and for $p \in \mathbb{Z}, x \in G_{\mathbb{N}}$, define

$$
\begin{aligned}
& \Psi_{p, p+1}(x)=x+\mathbf{e}(x) S_{p, p+1} \text { if } x \neq 0, \Psi_{p, p+1}(0)=\eta_{p} S_{p, p+1}^{+} . \\
& K_{p, p+1}(x)=\delta_{x+\mathbf{e}(x) S_{p, p+1}} \text { if } x \neq 0, K_{p, p+1}(0)=\sum_{i=1}^{N} \alpha_{i} \delta_{S_{p, p+1}^{+} \mathbf{e}_{i}} .
\end{aligned}
$$

In particular, we have $K_{p, p+1}(x)=E\left[\delta_{\Psi_{p, p+1}(x)} \mid \sigma(S)\right]$. Now we extend this definition for all $p \leq n \in \mathbb{Z}, x \in G_{\mathbb{N}}$ by setting

$$
\begin{gathered}
\Psi_{p, n}(x)=x 1_{\{p=n\}}+\Psi_{n-1, n} \circ \Psi_{n-2, n-1} \circ \cdots \circ \Psi_{p, p+1}(x) 1_{\{p>n\}}, \\
K_{p, n}(x)=\delta_{x} 1_{\{p=n\}}+K_{p, p+1} \cdots K_{n-2, n-1} K_{n-1, n}(x) 1_{\{p>n\}} .
\end{gathered}
$$

We equip $\mathscr{P}(G)$ with the following topology of weak convergence:

$$
\beta(P, Q)=\sup \left\{\left|\int g d P-\int g d Q\right|,\|g\|_{\infty}+\sup _{x \neq y} \frac{|g(x)-g(y)|}{|x-y|} \leq 1, g(0)=0\right\} .
$$

In this paper, starting from $(\Psi, K)$, we construct $\left(\varphi, K^{W}\right)$ and in particular show the following

Theorem 2. (1) $\Psi$ (resp. $K$ ) is a discrete flow of mappings (resp. kernels) on $G_{\mathbb{N}}$.

(2) There exists a joint realization $\left(\psi, N, \varphi, K^{W}\right)$ on a common probability space $(\Omega, \mathscr{A}, \mathbb{P})$ such that

(i) $(\psi, N) \stackrel{\text { law }}{=}(\Psi, K)$.

(ii) $(\varphi, W)$ (resp. $\left.\left(K^{W}, W\right)\right)$ is the unique flow of mappings (resp. Wiener flow) which solves $(T)$.

(iii) For all $s \in \mathbb{R}, T>0, x \in G, x_{n} \in \frac{1}{\sqrt{n}} G_{\mathbb{N}}$ such that $\lim _{n \rightarrow \infty} x_{n}=x$, we have

$$
\lim _{n \rightarrow \infty} \sup _{s \leq t \leq s+T}\left|\frac{1}{\sqrt{n}} \psi_{\lfloor n s\rfloor,\lfloor n t\rfloor}\left(\sqrt{n} x_{n}\right)-\varphi_{s, t}(x)\right|=0 \text { a.s. }
$$

and

$$
\lim _{n \rightarrow \infty} \sup _{s \leq t \leq s+T} \beta\left(K_{\lfloor n s\rfloor,\lfloor n t\rfloor}\left(\sqrt{n} x_{n}\right)(\sqrt{n} .), K_{s, t}^{W}(x)\right)=0 \text { a.s. }
$$

This theorem implies also the following

Corollary 1 For all $s \in \mathbb{R}, x \in G_{\mathbb{N}}$, let $t \longmapsto \Psi(t)$ be the linear interpolation of $\left(\Psi_{\lfloor n s\rfloor, k}(x), k \geq\lfloor n s\rfloor\right)$ and $\Psi_{s, t}^{n}(x):=\frac{1}{\sqrt{n}} \Psi(n t), K_{s, t}^{n}(x)=E\left[\delta_{\Psi_{s, t}^{n}(x)} \mid \sigma(S)\right], t \geq s, n \geq$ 1. For all $1 \leq p \leq q,\left(x_{i}\right)_{1 \leq i \leq q} \subset G$, let $x_{i}^{n} \in \frac{1}{\sqrt{n}} G_{\mathbb{N}}$ such that $\lim _{n \rightarrow \infty} x_{i}^{n}=x_{i}$. Define 


$$
Y^{n}=\left(\Psi_{s_{1}, \cdot}^{n}\left(\sqrt{n} x_{1}^{n}\right), \cdots, \Psi_{s_{p}, \cdot}^{n}\left(\sqrt{n} x_{p}^{n}\right), K_{s_{p+1}, \cdot}^{n}\left(\sqrt{n} x_{p+1}^{n}\right), \cdots, K_{s_{q}, \cdot}^{n}\left(\sqrt{n} x_{q}^{n}\right)\right) .
$$

Then

$$
Y^{n} \underset{n \rightarrow+\infty}{\stackrel{\text { law }}{\longrightarrow}} Y \text { in } \prod_{i=1}^{p} C\left(\left[s_{i},+\infty[, G) \times \prod_{j=p+1}^{q} C\left(\left[s_{j},+\infty[, \mathscr{P}(G))\right.\right.\right.\right.
$$

where

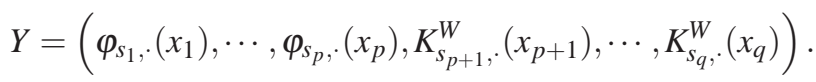

Our proof of Theorem 2 is based on a remarkable transformation introduced by Csaki and Vincze [9] which is strongly linked with Tanaka's SDE. Let $S$ be a simple random walk on $\mathbb{Z}(\mathrm{SRW})$ and $\varepsilon$ be a Bernoulli random variable independent of $S$ (just one!). Then there exists a SRW $M$ such that

$$
\sigma(M)=\sigma(\varepsilon, S)
$$

and moreover

$$
\left(\frac{1}{\sqrt{n}} S(n t), \frac{1}{\sqrt{n}} M(n t)\right)_{t \geq 0} \underset{n \rightarrow+\infty}{\stackrel{\text { law }}{\longrightarrow}}\left(B_{t}, W_{t}\right)_{t \geq 0} \text { in } C\left(\left[0, \infty\left[, \mathbb{R}^{2}\right) .\right.\right.
$$

where $t \longmapsto S(t)$ (resp. $M(t)$ ) is the linear interpolation of $S$ (resp. $M$ ) and $B, W$ are two Brownian motions satisying Tanaka's equation

$$
d W_{t}=\operatorname{sgn}\left(W_{t}\right) d B_{t}
$$

We will study this transformation with more details in Section 2 and then extend the result of Csaki and Vincze to Walsh Brownian motion (Proposition 2); Let $S=\left(S_{n}\right)_{n \in \mathbb{N}}$ be a SRW and associate to $S$ the process $Y_{n}:=S_{n}-\min _{k \leq n} S_{k}$, flip independently every "excursion "of $Y$ to each ray $D_{i}$ with probability $\alpha_{i}$, then the resulting process is not far from a random walk on $G$ whose law is given by (5). In Section 3, we prove Proposition 1 and study the scaling limits of $\Psi, K$.

\section{Csaki-Vincze transformation and consequences.}

In this section, we review a relevant result of Csaki and Vincze and then derive some useful consequences offering a better understanding of Tanaka's equation.

\subsection{Csaki-Vincze transformation.}

Theorem 3. ([9] page 109) Let $S=\left(S_{n}\right)_{n \geq 0}$ be a SRW. Then, there exists a $S R W$ $\bar{S}=\left(\bar{S}_{n}\right)_{n \geq 0}$ such that: 


$$
\bar{Y}_{n}:=\max _{k \leq n} \bar{S}_{k}-\bar{S}_{n} \Rightarrow\left|\bar{Y}_{n}-\right| S_{n}|| \leq 2 \forall n \in \mathbb{N} .
$$

Sketch of the proof. Here, we just give the expression of $\bar{S}$ with some useful comments (see also the figures below). We insist that a careful reading of the pages 109 and 110 [9] is recommended for the sequel. Let $X_{i}=S_{i}-S_{i-1}, i \geq 1$ and define

$$
\tau_{1}=\min \left\{i>0: S_{i-1} S_{i+1}<0\right\}, \tau_{l+1}=\min \left\{i>\tau_{l}: S_{i-1} S_{i+1}<0\right\} \forall l \geq 1 .
$$

For $j \geq 1$, set

$$
\bar{X}_{j}=\sum_{l \geq 0}(-1)^{l+1} X_{1} X_{j+1} 1_{\left\{\tau_{l}+1 \leq j \leq \tau_{l+1}\right\}} .
$$

Let $\bar{S}_{0}=0, \bar{S}_{j}=\bar{X}_{1}+\cdots+\bar{X}_{j}, j \geq 1$. Then, the theorem holds for $\bar{S}$. We call $T(S)=\bar{S}$ the Csaki-Vincze transformation of $S$.

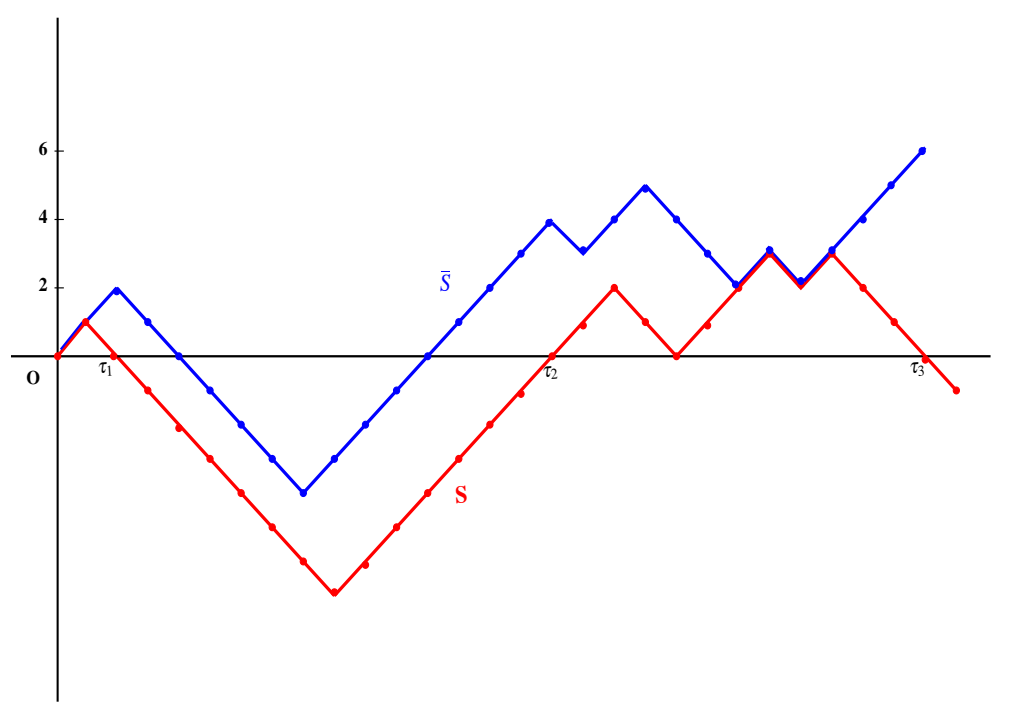

Fig. $2 S$ and $\bar{S}$.

Note that $T$ is an even function, that is $T(S)=T(-S)$. As a consequence of $(i i i)$ and (iv) [9] (page 110), we have

$$
\tau_{l}=\min \left\{n \geq 0, \bar{S}_{n}=2 l\right\} \forall l \geq 1 .
$$

This entails the following

Corollary 2 (1) Let $S$ be a SRW and define $\bar{S}=T(S)$. Then

(i) For all $n \geq 0$, we have $\sigma\left(\bar{S}_{j}, j \leq n\right) \vee \sigma\left(S_{1}\right)=\sigma\left(S_{j}, j \leq n+1\right)$. 


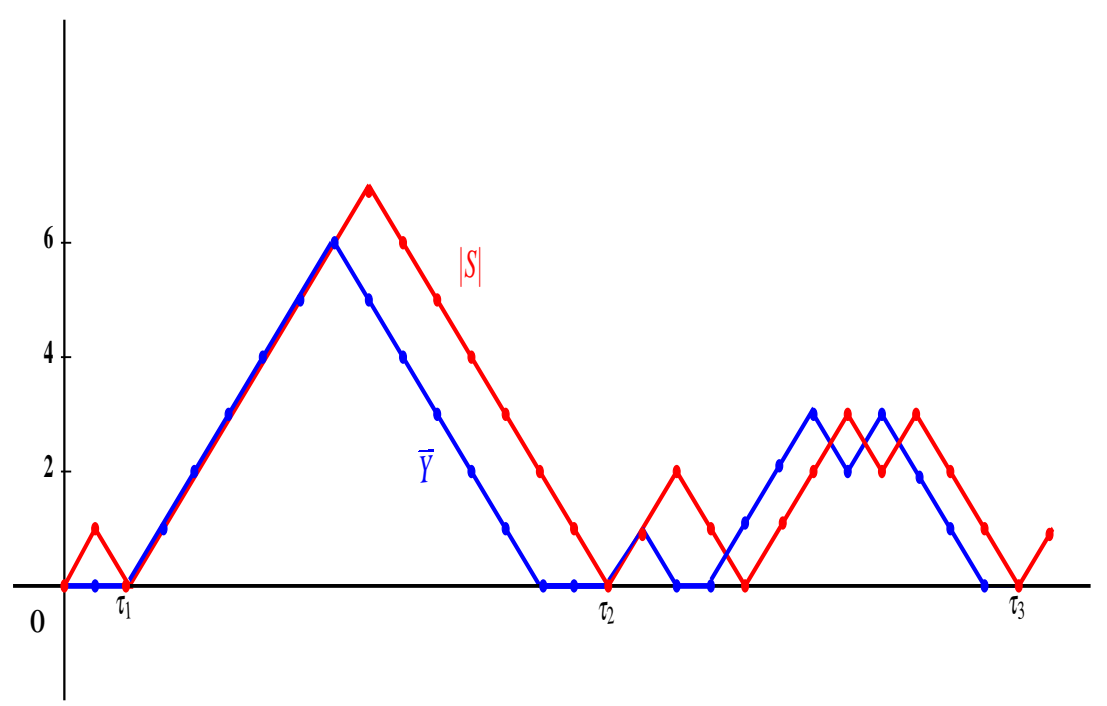

Fig. $3|S|$ and $\bar{Y}$.

(ii) $S_{1}$ is independent of $\sigma(\bar{S})$.

(2) Let $\bar{S}=\left(\bar{S}_{k}\right)_{k \geq 0}$ be a SRW. Then

(i) There exists a SRW S such that:

$$
\bar{Y}_{n}:=\max _{k \leq n} \bar{S}_{k}-\bar{S}_{n} \Rightarrow\left|\bar{Y}_{n}-\right| S_{n}|| \leq 2 \forall n \in \mathbb{N} .
$$

(ii) $T^{-1}\{\bar{S}\}$ is reduced to exactly two elements $S$ and $-S$ where $S$ is obtained by adding information to $\bar{S}$.

Proof. (1) We retain the notations just before the corollary. (i) To prove the inclusion $\subset$, we only need to check that $\left\{\tau_{l}+1 \leq j \leq \tau_{l+1}\right\} \in \sigma\left(S_{h}, h \leq n+1\right)$ for a fixed $j \leq n$. This is clear since $\left\{\tau_{l}=m\right\} \in \sigma\left(S_{h}, h \leq m+1\right)$ for all $l, m \in \mathbb{N}$. For all $1 \leq j \leq n$, we have $X_{j+1}=\sum_{l \geq 0}(-1)^{l+1} X_{1} \bar{X}_{j} 1_{\left\{\tau_{l}+1 \leq j \leq \tau_{l+1}\right\}}$. By (7), $\left\{\tau_{l}+1 \leq j \leq\right.$ $\left.\tau_{l+1}\right\} \in \sigma\left(\bar{S}_{h}, h \leq j-1\right)$ and so the inclusion $\supset$ holds. (ii) We may write

$\tau_{1}=\min \left\{i>1: X_{1} S_{i-1} X_{1} S_{i+1}<0\right\}, \tau_{l+1}=\min \left\{i>\tau_{l}: X_{1} S_{i-1} X_{1} S_{i+1}<0\right\} \forall l \geq 1$

This shows that $\bar{S}$ is $\sigma\left(X_{1} X_{j+1}, j \geq 0\right)$-measurable and (ii) is proved.

(2) (i) Set $\bar{X}_{j}=\bar{S}_{j}-\bar{S}_{j-1}, j \geq 1$ and $\tau_{l}=\min \left\{n \geq 0, \bar{S}_{n}=2 l\right\}$ for all $l \geq 1$. Let $\varepsilon$ be a random variable independent of $\bar{S}$ such that:

$$
\mathbb{P}(\varepsilon=1)=\mathbb{P}(\varepsilon=-1)=\frac{1}{2} .
$$

Define 


$$
X_{j+1}=\varepsilon 1_{\{j=0\}}+\left(\sum_{l \geq 0}(-1)^{l+1} \varepsilon \bar{X}_{j} 1_{\left\{\tau_{l}+1 \leq j \leq \tau_{l+1}\right\}}\right) 1_{\{j \geq 1\}} .
$$

Then set $S_{0}=0, S_{j}=X_{1}+\cdots X_{j}, j \geq 1$. It is not hard to see that the sequence of the random times $\tau_{i}(S), i \geq 1$ defined from $S$ as in Theorem 3 is exactly $\tau_{i}, i \geq 1$ and therefore $T(S)=\bar{S}$. (ii) Let $S$ such that $T(S)=\bar{S}$. By (1), $\sigma(\bar{S}) \vee \sigma\left(S_{1}\right)=\sigma(S)$ and $S_{1}$ is independent of $\bar{S}$ which proves (ii).

\subsection{The link with Tanaka's equation.}

Let $S$ be a SRW, $\bar{S}=-T(S)$ and $t \longmapsto S(t)$ (resp. $\bar{S}(t))$ be the linear interpolation of $S\left(\right.$ resp. $\bar{S}$ ) on $\mathbb{R}$. Define for all $n \geq 1, S_{t}^{(n)}=\frac{1}{\sqrt{n}} S(n t), \bar{S}_{t}^{(n)}=\frac{1}{\sqrt{n}} \bar{S}(n t)$. Then, it can be easily checked (see Proposition 2.4 in [3] page 107) that

$$
\left(\bar{S}_{t}^{(n)}, S_{t}^{(n)}\right)_{t \geq 0} \underset{n \rightarrow+\infty}{\stackrel{\text { law }}{\longrightarrow}}\left(B_{t}, W_{t}\right)_{t \geq 0} \text { in } C\left(\left[0, \infty\left[, \mathbb{R}^{2}\right) .\right.\right.
$$

In particular $B$ and $W$ are two standard Brownian motions. On the other hand, $\mid Y_{n}^{+}-$ $\mid S_{n} \| \leq 2 \forall n \in \mathbb{N}$ with $Y_{n}^{+}:=\bar{S}_{n}-\min _{k \leq n} \bar{S}_{k}$ by Theorem 3 which implies $\left|W_{t}\right|=B_{t}-$ $\min _{0 \leq u \leq t} B_{u}$. Tanaka's formula for local time gives

$$
\left|W_{t}\right|=\int_{0}^{t} \operatorname{sgn}\left(W_{u}\right) d W_{u}+L_{t}(W)=B_{t}-\min _{0 \leq u \leq t} B_{u},
$$

where $L_{t}(W)$ is the local time at 0 of $W$ and so

$$
d W_{u}=\operatorname{sgn}\left(W_{u}\right) d B_{u} .
$$

We deduce that for each SRW $S$ the couple $(-T(S), S)$, suitably normalized and time scaled converges in law towards $(B, W)$ satisfying (8). Finally, remark that $-T(S)=\bar{S} \Rightarrow-T(-S)=\bar{S}$ is the analogue of $W$ solves $(8) \Rightarrow-W$ solves $(8)$. We have seen how to construct solutions to (8) by means of $T$. In the sequel, we will use this approach to construct a stochastic flow of mappings which solves equation $(T)$ in general.

\subsection{Extensions.}

Let $S=\left(S_{n}\right)_{n \geq 0}$ be a SRW and set $Y_{n}:=\max _{k \leq n} S_{k}-S_{n}$. For $0 \leq p<q$, we say that $E=[p, q]$ is an excursion for $Y$ if the following conditions are satisfied (with the convention $Y_{-1}=0$ ):

- $Y_{p}=Y_{p-1}=Y_{q}=Y_{q+1}=0$. 
- $\forall p \leq j<q, Y_{j}=0 \Rightarrow Y_{j+1}=1$.

For example in Figure 3, $[2,14],[16,18]$ are excursions for $\bar{Y}$. If $E=[p, q]$ is an excursion for $Y$, define $e(E):=p, f(E):=q$.

Let $\left(E_{i}\right)_{i \geq 1}$ be the random set of all excursions of $Y$ ordered such that: $e\left(E_{i}\right)<$ $e\left(E_{j}\right) \forall i<j$. From now on, we call $E_{i}$ the $i$ th excursion of $Y$. Then, we have

Proposition 2 On a probability space $(\Omega, \mathscr{A}, P)$, consider the following jointly independent processes:

- $\eta=\left(\eta_{i}\right)_{i \geq 1}$, a sequence of i.i.d random variables distributed according to $\sum_{i=1}^{N} \alpha_{i} \delta_{\mathbf{e}_{i}}$

- $\left(\bar{S}_{n}\right)_{n \in \mathbb{N}} a S R W$.

Then, on an extension of $(\Omega, \mathscr{A}, P)$, there exists a Markov chain $\left(M_{n}\right)_{n \in \mathbb{N}}$ started at 0 with stochastic matrix given by (5) such that:

$$
\bar{Y}_{n}:=\max _{k \leq n} \bar{S}_{k}-\bar{S}_{n} \Rightarrow\left|M_{n}-\eta_{i} \bar{Y}_{n}\right| \leq 2
$$

on the ith excursion of $\bar{Y}$.

Proof. Fix $S \in T^{-1}\{\bar{S}\}$. Then, by Corollary 2, we have $\left|\bar{Y}_{n}-\right| S_{n}|| \leq 2 \forall n \in \mathbb{N}$. Consider a sequence $\left(\beta_{i}\right)_{i \geq 1}$ of i.i.d random variables distributed according to $\sum_{i=1}^{N} \alpha_{i} \delta_{\mathbf{e}_{i}}$ which is independent of $(\bar{S}, \eta)$. Denote by $\left(\tau_{l}\right)_{l \geq 1}$ the sequence of random times constructed in the proof of Theorem 3 from $S$. It is sufficient to look to what happens at each interval $\left[\tau_{l}, \tau_{l+1}\right]$ (with the convention $\tau_{0}=0$ ).

Using (7), we see that in $\left[\tau_{l}, \tau_{l+1}\right]$ there are two jumps of $\max _{k \leq n} \bar{S}_{k}$; from $2 l$ to $2 l+1$

$\left(J_{1}\right)$ and from $2 l+1$ to $2 l+2\left(J_{2}\right)$. The last jump $\left(J_{2}\right)$ occurs always at $\tau_{l+1}$ by (7). Consequently there are only 3 possible cases:

(i) There is no excursion of $\bar{Y}\left(J_{1}\right.$ and $J_{2}$ occur respectively at $\tau_{l}+1$ and $\tau_{l}+2$, see $\left[0, \tau_{1}\right]$ in Figure 3).

(ii) There is just one excursion of $\bar{Y}$ (see $\left[\tau_{1}, \tau_{2}\right]$ in Figure 3 ).

(iii) There are 2 excursions of $\bar{Y}$ (see $\left[\tau_{2}, \tau_{3}\right]$ in Figure 3 ).

Note that: $\bar{Y}_{\tau_{l}}=\bar{Y}_{\tau_{l+1}}=S_{\tau_{l}}=S_{\tau_{l+1}}=0$. In the case (i), we have necessarily $\tau_{l+1}=\tau_{l}+2$. Set $M_{n}=\beta_{l} \cdot\left|S_{n}\right| \quad \forall n \in\left[\tau_{l}, \tau_{l+1}\right]$.

To treat other cases, the following remarks may be useful: from the expression of $\bar{S}$, we have $\forall l \geq 0$

(a)If $k \in\left[\tau_{l}+2, \tau_{l+1}\right], \bar{S}_{k-1}=2 l+1 \Longleftrightarrow S_{k}=0$.

(b)If $k \in\left[\tau_{l}, \tau_{l+1}\right], \bar{Y}_{k}=0 \Rightarrow\left|S_{k+1}\right| \in\{0,1\}$ and $S_{k+1}=0 \Rightarrow \bar{Y}_{k}=0$.

In the case (ii), let $E_{l}^{1}$ be the unique excursion of $\bar{Y}$ in the interval $\left[\tau_{l}, \tau_{l+1}\right]$. Then, we have two subcases:

(ii1) $f\left(E_{l}^{1}\right)=\tau_{l+1}-2\left(J_{1}\right.$ occurs at $\left.\tau_{l+1}-1\right)$.

If $\tau_{l}+2 \leq k \leqslant f\left(E_{l}^{1}\right)+1$, then $k-1 \leqslant f\left(E_{l}^{1}\right)$, and so $\bar{S}_{k-1} \neq 2 l+1$. Using (a), we get: $S_{k} \neq 0$. Thus, in this case the first zero of $S$ after $\tau_{l}$ is $\tau_{l+1}$. Set: $M_{n}=\eta_{N\left(E_{l}^{1}\right)}$. $\left|S_{n}\right|$, 
where $N(E)$ is the number of the excursion $E$.

(ii2) $f\left(E_{l}^{1}\right)=\tau_{l+1}-1\left(J_{1}\right.$ occurs at $\tau_{l}+1$ and so $\left.\left.\bar{Y}_{\tau_{l}+1}=0\right)\right)$. In this case, using (b) and the figure below we see that the first zero $\tau_{l}^{*}$ of $S$ after $\tau_{l}$ is $e\left(E_{l}^{1}\right)+1=\tau_{l}+2$.

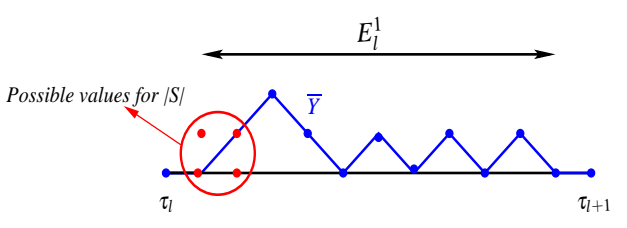

Fig. 4 The case (ii2).

Set

$$
M_{n}= \begin{cases}\beta_{l} \cdot\left|S_{n}\right| & \text { if } n \in\left[\tau_{l}, \tau_{l}^{*}-1\right] \\ \eta_{N\left(E_{l}^{1}\right)} \cdot\left|S_{n}\right| & \text { if } n \in\left[\tau_{l}^{*}, \tau_{l+1}\right]\end{cases}
$$

In the case (iii), let $E_{l}^{1}$ and $E_{l}^{2}$ denote respectively the first and 2nd excursion of $\bar{Y}$ in $\left[\tau_{l}, \tau_{l+1}\right]$. We have, $\tau_{l}+2 \leq k \leq e\left(E_{l}^{2}\right) \Rightarrow k-1 \leq e\left(E_{l}^{2}\right)-1=f\left(E_{l}^{1}\right) \Rightarrow \bar{S}_{k-1} \neq$ $2 l+1 \Rightarrow S_{k} \neq 0$ by (a). Hence, the first zero of $S$ after $\tau_{l}$ is $\tau_{l}^{*}:=e\left(E_{l}^{2}\right)+1$ using $\bar{Y}_{k}=0 \Rightarrow\left|S_{k+1}\right| \in\{0,1\}$ in (b). Set:

$$
M_{n}= \begin{cases}\eta_{N\left(E_{l}^{1}\right)} \cdot\left|S_{n}\right| & \text { if } n \in\left[\tau_{l}, \tau_{l}^{*}-1\right] \\ \eta_{N\left(E_{l}^{2}\right) \cdot \mid} \cdot S_{n} \mid & \text { if } n \in\left[\tau_{l}^{*}, \tau_{l+1}\right]\end{cases}
$$

Let $\left(M_{n}\right)_{n \in \mathbb{N}}$ be the process constructed above. Then clearly $\left|M_{n}-\eta_{i} \bar{Y}_{n}\right| \leq 2$ on the $i$ th excursion of $\bar{Y}$.

To complete the proof, it suffices to show that the law of $\left(M_{n}\right)_{n \in \mathbb{N}}$ is given by (5). The only point to verify is $\mathbb{P}\left(M_{n+1}=\mathbf{e}_{i} \mid M_{n}=0\right)=\alpha_{i}$. For this, consider on another probability space the jointly independent processes $(S, \gamma, \lambda)$ such that $S$ is a SRW and $\gamma, \lambda$ have the same law as $\eta$. Let $\left(\tau_{l}\right)_{l \geq 1}$ be the sequence of random times defined from $S$ as in Theorem 3. For all $l \in \mathbb{N}$, denote by $\tau_{l}^{*}$ the first zero of $S$ after $\tau_{l}$ and set

$$
V_{n}= \begin{cases}\gamma_{l} \cdot\left|S_{n}\right| & \text { if } n \in\left[\tau_{l}, \tau_{l}^{*}-1\right] \\ \lambda_{l} \cdot\left|S_{n}\right| & \text { if } n \in\left[\tau_{l}^{*}, \tau_{l+1}\right]\end{cases}
$$

It is clear, by construction, that $M \stackrel{\text { law }}{=} V$. We can write:

$$
\left\{\tau_{0}, \tau_{0}^{*}, \tau_{1}, \tau_{1}^{*}, \tau_{2}, \cdots\right\}=\left\{T_{0}, T_{1}, T_{2}, \cdots\right\} \text { with } T_{0}=0<T_{1}<T_{2}<\cdots .
$$


For all $k \geq 0$, let $\zeta_{k}:=\sum_{j=0}^{N} \mathbf{e}_{j} 1_{\left\{\left.V\right|_{\left[T_{k}, T_{k+1}\right]} \in D_{j}\right\}}$. Obviously, $S$ and $\zeta_{k}$ are independent and $\zeta_{k} \stackrel{\text { law }}{=} \sum_{i=1}^{N} \alpha_{i} \delta_{\mathbf{e}_{i}}$. Furthermore

$$
\begin{aligned}
\mathbb{P}\left(V_{n+1}=\mathbf{e}_{i} \mid V_{n}=0\right) & =\frac{1}{\mathbb{P}\left(S_{n}=0\right)} \sum_{k=0}^{+\infty} \mathbb{P}\left(V_{n+1}=\mathbf{e}_{i}, S_{n}=0, n \in\left[T_{k}, T_{k+1}[)\right.\right. \\
& =\frac{1}{\mathbb{P}\left(S_{n}=0\right)} \sum_{k=0}^{+\infty} \mathbb{P}\left(\zeta_{k}=\mathbf{e}_{i}, S_{n}=0, n \in\left[T_{k}, T_{k+1}[)\right.\right. \\
& =\alpha_{i}
\end{aligned}
$$

This completes the proof of the proposition.

Remark 1 With the notations of Proposition 2, let $(\eta \cdot \bar{Y})$ be the Markov chain defined by $(\eta \cdot \bar{Y})_{n}=\eta_{i} \bar{Y}_{n}$ on the ith excursion of $\bar{Y}$ and $(\eta \cdot \bar{Y})_{n}=0$ if $\bar{Y}_{n}=0$. Then the stochastic Matrix of $(\eta \cdot \bar{Y})$ is given by

$M(0,0)=\frac{1}{2}, M\left(0, \mathbf{e}_{i}\right)=\frac{\alpha_{i}}{2}, M\left(n \mathbf{e}_{i},(n+1) \mathbf{e}_{i}\right)=M\left(n \mathbf{e}_{i},(n-1) \mathbf{e}_{i}\right)=\frac{1}{2}, i \in[1, N], n \in \mathbb{N}^{*}$.

\section{Proof of main results.}

\subsection{Proof of Proposition 1.}

Let $\left(Z_{t}\right)_{t \geq 0}$ be a $W\left(\alpha_{1}, \cdots, \alpha_{N}\right)$ on $G$ started at 0 . For all $i \in[1, N]$, define $Z_{t}^{i}=$ $\left|Z_{t}\right| 1_{\left\{Z_{t} \in D_{i}\right\}}-\left|Z_{t}\right| 1_{\left\{Z_{t} \notin D_{i}\right\}}$. Then $Z_{t}^{i}=\Phi^{i}\left(Z_{t}\right)$ where $\Phi^{i}(x)=|x| 1_{\left\{x \in D_{i}\right\}}-|x| 1_{\left\{x \notin D_{i}\right\}}$. Let $Q^{i}$ be the semigroup of the skew Brownian motion of parameter $\alpha_{i}\left(S B M\left(\alpha_{i}\right)\right)$ (see [10] page 87). Then the following relation is easy to check: $P_{t}\left(f \circ \Phi^{i}\right)=Q_{t}^{i} f \circ \Phi^{i}$ for all bounded measurable function $f$ defined on $\mathbb{R}$. This shows that $Z^{i}$ is a $\operatorname{SBM}\left(\alpha_{i}\right)$ started at 0 . For $n \geq 1, i \in[1, N]$, define

$$
\begin{gathered}
T_{0}^{n}=0, \quad T_{k+1}^{n}=\inf \left\{r \geq 0: d\left(Z_{r}, Z_{T_{k}^{n}}\right)=\frac{1}{\sqrt{n}}\right\}, k \geq 0 . \\
T_{0}^{n, i}=0, \quad T_{k+1}^{n, i}=\inf \left\{r \geq 0:\left|Z_{r}^{i}-Z_{T_{k}^{n, i}}^{i}\right|=\frac{1}{\sqrt{n}}\right\}, k \geq 0 .
\end{gathered}
$$

Remark that $T_{k+1}^{n}=T_{k+1}^{n, i}=\inf \left\{r \geq 0:|| Z_{r}|-| Z_{T_{k}^{n}}||=\frac{1}{\sqrt{n}}\right\}$. Furthermore if $Z_{t} \in D_{i}$, then obviously $d\left(Z_{t}, Z_{s}\right)=\left|Z_{t}^{i}-Z_{s}^{i}\right|$ for all $s \geq 0$ and consequently 


$$
d\left(Z_{t}, Z_{s}\right) \leq \sum_{i=1}^{N}\left|Z_{t}^{i}-Z_{s}^{i}\right|
$$

Now define $Z_{k}^{n}=\sqrt{n} Z_{T_{k}^{n}}, Z_{k}^{n, i}=\sqrt{n} Z_{T_{k}^{n, i}}^{i}$. Then $\left(Z_{k}^{n}, k \geq 0\right) \stackrel{\text { law }}{=} M$ (see the proof of Proposition 2 in [5]). For all $T>0$, we have

$$
\sup _{t \in[0, T]} d\left(Z_{t}, \frac{1}{\sqrt{n}} Z_{\lfloor n t\rfloor}^{n}\right) \leq \sum_{i=1}^{N} \sup _{t \in[0, T]}\left|Z_{t}^{i}-\frac{1}{\sqrt{n}} Z_{\lfloor n t\rfloor}^{n, i}\right| \underset{n \rightarrow+\infty}{\longrightarrow} 0 \text { in probability }
$$

by Lemma 4.4 [1] which proves our result.

Remarks 1 (1) By (10), a.s. $t \mapsto Z_{t}$ is continuous. We will always suppose that Walsh Brownian motion is continuous.

(2) By combining the two propositions 1 and 2, we deduce that $(\eta \cdot \bar{Y})$ rescales as Walsh Brownian motion in the space of continuous functions. It is also possible to prove this result by showing that the family of laws is tight and that any limit process along a subsequence is the Walsh Brownian motion.

\subsection{Scaling limits of $(\Psi, K)$.}

Set $\eta_{p, n}=\mathbf{e}\left(\Psi_{p, n}\right)$ for all $p \leq n$ where $\Psi_{p, n}=\Psi_{p, n}(0)$.

Proposition 3 (i) For all $p \leq n,\left|\Psi_{p, n}\right|=S_{p, n}^{+}$.

(ii) For all $p<n<q$,

$$
\mathbb{P}\left(\eta_{p, q}=\eta_{n, q} \mid \min _{h \in[p, q]} S_{h}=\min _{h \in[n, q]} S_{h}\right)=1
$$

and

$$
\mathbb{P}\left(\eta_{p, n}=\eta_{p, q} \mid \min _{h \in[p, n]} S_{h}=\min _{h \in[p, q]} S_{h}, S_{p, j}^{+}>0 \forall j \in[n, q]\right)=1 .
$$

(iii) $\operatorname{Set} T_{p, x}=\inf \left\{q \geq p: S_{q}-S_{p}=-|x|\right\}$. Then for all $p \leq n, x \in G_{\mathbb{N}}$,

$$
\begin{gathered}
\Psi_{p, n}(x)=\left(x+\mathbf{e}(x) S_{p, n}\right) 1_{\left\{n \leq T_{p, x}\right\}}+\Psi_{p, n} 1_{\left\{n>T_{p, x}\right\}} ; \\
K_{p, n}(x)=E\left[\delta_{\Psi_{p, n}(x)} \mid \sigma(S)\right]=\delta_{x+\mathbf{e}(x) S_{p, n}} 1_{\left\{n \leq T_{p, x}\right\}}+\sum_{i=1}^{N} \alpha_{i} \delta_{S_{p, n}^{+} \mathbf{e}_{i}} 1_{\left\{n>T_{p, x}\right\}} .
\end{gathered}
$$

Proof. (i) We take $p=0$ and prove the result by induction on $n$. For $n=0$, this is clear. Suppose the result holds for $n$. If $\Psi_{0, n} \in G^{*}$, then $S_{0, n}^{+}>0$ and so $\min _{h \in[0, n]} S_{h}=$ $\min _{h \in[0, n+1]} S_{h}$. Moreover $\Psi_{0, n+1}=\Psi_{0, n}+\eta_{0, n} S_{n, n+1}=\left(S_{n+1}-\min _{h \in[0, n]} S_{h}\right) \eta_{0, n}=$ $S_{0, n+1}^{+} \eta_{0, n}$. If $\Psi_{0, n}=0$, then $S_{0, n}^{+}=0$ and $\left|\Psi_{0, n+1}\right|=S_{n, n+1}^{+}$. But $\min _{h \in[0, n+1]} S_{h}=$ $\min \left(\min _{h \in[0, n]} S_{h}, S_{n+1}\right)=\min \left(S_{n}, S_{n+1}\right)$ since $S_{0, n}^{+}=0$ which proves (i). 
(ii) Let $p<n<q$. If $\min _{h \in[p, q]} S_{h}=\min _{h \in[n, q]} S_{h}$, then $S_{p, q}^{+}=S_{n, q}^{+}$. When $S_{p, q}^{+}=0$, we have $\eta_{p, q}=\eta_{n, q}=\mathbf{e}_{N}$ by convention. Suppose that $S_{p, q}^{+}>0$, then clearly

$$
J:=\sup \left\{j<q: S_{p, j}^{+}=0\right\}=\sup \left\{j<q: S_{n, j}^{+}=0\right\} .
$$

By the flow property of $\Psi$, we have $\Psi_{p, q}=\Psi_{n, q}=\Psi_{J, q}$. The second assertion of (ii) is also clear.

(iii) By (i), we have $\Psi_{p, n}=\Psi_{p, n}(x)=0$ if $n=T_{p, x}$ and so $\Psi_{p, .}(x)$ is given by $\Psi_{p, \text {. }}$ after $T_{p, x}$ using the cocyle property. The last claim is easy to establish.

For all $s \in \mathbb{R}$, let $d_{s}$ (resp. $d_{\infty}$ ) be the distance of uniform convergence on every compact subset of $C\left(\left[s,+\infty[, G)(\right.\right.$ resp. $C(\mathbb{R}, \mathbb{R}))$. Denote by $\mathbb{D}=\left\{s_{n}, n \in \mathbb{N}\right\}$ the set of all dyadic numbers of $\mathbb{R}$ and define $\widetilde{C}=C(\mathbb{R}, \mathbb{R}) \times \prod_{n=0}^{+\infty} C\left(\left[s_{n},+\infty[, G)\right.\right.$ equipped with the metric:

$d(x, y)=d_{\infty}\left(x^{\prime}, y^{\prime}\right)+\sum_{n=0}^{+\infty} \frac{1}{2^{n}} \inf \left(1, d_{s_{n}}\left(x_{n}, y_{n}\right)\right)$ where $x=\left(x^{\prime}, x_{s_{0}}, \cdots\right), y=\left(y^{\prime}, y_{s_{0}}, \cdots\right)$.

Let $t \longmapsto S(t)$ be the linear interpolation of $S$ on $\mathbb{R}$ and define $S_{t}^{(n)}=\frac{1}{\sqrt{n}} S(n t), n \geq 1$. If $u \leq 0$, we define $\lfloor u\rfloor=-\lfloor-u\rfloor$. Then, we have

$$
S_{t}^{(n)}=S_{t}^{n}+o\left(\frac{1}{\sqrt{n}}\right) \text {, with } S_{t}^{n}:=\frac{1}{\sqrt{n}} S_{\lfloor n t\rfloor} .
$$

Let $\Psi_{s, t}^{n}=\Psi_{s, t}^{n}(0)$ (defined in Corollary 1). Then $\Psi_{s, t}^{(n)}:=\frac{1}{\sqrt{n}} \Psi_{\lfloor n s\rfloor,\lfloor n t\rfloor}+o\left(\frac{1}{\sqrt{n}}\right)$ and we have the following

Lemma 1. Let $\mathbb{P}_{n}$ be the law of $Z^{n}=\left(S^{(n)},\left(\Psi_{s_{i},}^{(n)}\right)_{i \in \mathbb{N}}\right)$ in $\widetilde{C}$. Then $\left(\mathbb{P}_{n}, n \geq 1\right)$ is tight.

Proof. By Donsker theorem $\mathbb{P}_{S^{(n)}} \longrightarrow \mathbb{P}_{W}$ in $C(\mathbb{R}, \mathbb{R})$ as $n \rightarrow \infty$ where $\mathbb{P}_{W}$ is the law of any Brownian motion on $\mathbb{R}$. Let $\mathbb{P}_{Z_{s_{i}}}$ be the law of any $W\left(\alpha_{1}, \cdots, \alpha_{N}\right)$ started at 0 at time $s_{i}$. Plainly, the law of $\Psi_{p, p+}$. is given by (9) and so by Propositions 1 and 2, for all $i \in \mathbb{N}, \mathbb{P}_{\Psi_{s_{i} . .}^{(n)}} \longrightarrow \mathbb{P}_{Z_{s_{i}}}$ in $C\left(\left[s_{i},+\infty[, G)\right.\right.$ as $n \rightarrow \infty$. Now the lemma holds using Proposition 2.4 [3] (page 107).

Fix a sequence $\left(n_{k}, k \in \mathbb{N}\right)$ such that $Z^{n_{k}} \underset{k \rightarrow+\infty}{\stackrel{\text { law }}{\longrightarrow}} Z$ in $\widetilde{C}$. In the next paragraph, we will describe the law of $Z$. Notice that $\left(\Psi_{p, n}\right)_{p \leq n}$ and $S$ can be recovered from

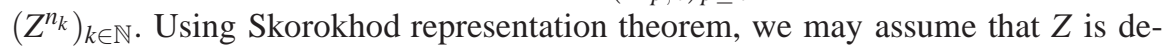
fined on the original probability space and the preceding convergence holds almost surely. Write $Z=\left(W, \psi_{s_{1},}, \psi_{s_{2},}, \cdots\right)$. Then, $\left(W_{t}\right)_{t \in \mathbb{R}}$ is a Brownian motion on $\mathbb{R}$ and $\left(\psi_{s, t}\right)_{t \geq s}$ is an $W\left(\alpha_{1}, \cdots, \alpha_{N}\right)$ started at 0 for all $s \in \mathbb{D}$. 


\subsubsection{Description of the limit process.}

Set $\gamma_{s, t}=\mathbf{e}\left(\psi_{s, t}\right), s \in \mathbb{D}, s<t$ and define $\min _{u, v}=\min _{r \in[u, v]} W_{r}, u \leq v \in \mathbb{R}$. Then, we have

Proposition 4 (i) For all $s \leq t, s \in \mathbb{D}$, $\left|\psi_{s, t}\right|=W_{s, t}^{+}$.

(ii) For all $s<t, u<v, s, u \in \mathbb{D}$,

$$
\mathbb{P}\left(\gamma_{s, t}=\gamma_{u, v} \mid \min _{s, t}=\min _{u, v}\right)=1 \text { if } \mathbb{P}\left(\min _{s, t}=\min _{u, v}\right)>0 .
$$

Proof. (i) is immediate from the convergence of $Z^{n_{k}}$ towards $Z$ and Proposition 3 (i). (ii) We first prove that for all $s<t<u$,

$$
\mathbb{P}\left(\gamma_{s, u}=\gamma_{t, u} \mid \min _{s, u}=\min _{t, u}\right)=1 \text { if } s, t \in \mathbb{D}
$$

and

$$
\mathbb{P}\left(\gamma_{s, t}=\gamma_{s, u} \mid \min _{s, t}=\min _{s, u}\right)=1 \text { if } s \in \mathbb{D} .
$$

Fix $s<t<u$ with $s, t \in \mathbb{D}$ and let show that a.s.

$$
\left\{\min _{s, u}=\min _{t, u}\right\} \subset\left\{\exists k_{0}, \quad \eta_{\left\lfloor n_{k} s\right\rfloor,\left\lfloor n_{k} u\right\rfloor}=\eta_{\left.\left\lfloor n_{k}\right\rfloor\right\rfloor,\left\lfloor n_{k} u\right\rfloor} \text { for all } k \geq k_{0}\right\} .
$$

We have $\left\{\min _{s, u}=\min _{t, u}\right\}=\left\{\min _{s, t}<\min _{t, u}\right\}$ a.s. By uniform convergence the last set is contained in

$$
\left\{\exists k_{0}, \min _{\left\lfloor n_{k} s\right\rfloor \leq j \leq\left\lfloor n_{k} t\right\rfloor} S_{j}<\min _{\left\lfloor n_{k} t\right\rfloor \leq j \leq\left\lfloor n_{k} u\right\rfloor} S_{j} \text { for all } k \geq k_{0}\right\}
$$

which is a subset of

$$
\left\{\exists k_{0}, \min _{\left\lfloor n_{k} s\right\rfloor \leq j \leq\left\lfloor n_{k} u\right\rfloor} S_{j}=\min _{\left\lfloor n_{k}\right\rfloor \leq j \leq\left\lfloor n_{k} u\right\rfloor} S_{j} \text { for all } k \geq k_{0}\right\} .
$$

This gives (13) using Proposition 3 (ii). Since $x \longrightarrow \mathbf{e}(x)$ is continuous on $G^{*}$, on $\left\{\min _{s, u}=\min _{t, u}\right\}$, we have

$$
\gamma_{s, u}=\lim _{k \rightarrow \infty} \mathbf{e}\left(\frac{1}{\sqrt{n_{k}}} \Psi_{\left\lfloor n_{k} s\right\rfloor,\left\lfloor n_{k} u\right\rfloor}\right)=\lim _{k \rightarrow \infty} \mathbf{e}\left(\frac{1}{\sqrt{n}_{k}} \Psi_{\left\lfloor n_{k} t\right\rfloor,\left\lfloor n_{k} u\right\rfloor}\right)=\gamma_{t, u} \text { a.s. }
$$

which proves (11). If $s \in \mathbb{D}, t>s$ and $\min _{s, t}=\min _{s, u}$, then $s$ and $t$ are in the same excursion interval of $W_{s}^{+}$and so $W_{s, r}^{+}>0$ for all $r \in[t, u]$. As preceded, $\left\{\min _{s, t}=\right.$ $\left.\min _{s, u}\right\}$ is a.s. included in

$$
\left\{\exists k_{0}, \min _{\left\lfloor n_{k} s\right\rfloor \leq j \leq\left\lfloor n_{k} t\right\rfloor} S_{j}=\min _{\left\lfloor n_{k} s\right\rfloor \leq j \leq\left\lfloor n_{k} u\right\rfloor} S_{j}, S_{\left\lfloor n_{k} s\right\rfloor, j}^{+}>0 \forall j \in\left[\left\lfloor n_{k} t\right\rfloor,\left\lfloor n_{k} u\right\rfloor\right], k \geq k_{0}\right\} .
$$

Now it is easy to deduce (12) using Proposition 3 (ii). To prove (ii), suppose that $s \leq u, \min _{s, t}=\min _{u, v}$. There are two cases to discuss, (a) $s \leq u \leq v \leq t$, (b) $s \leq$ $u \leq t \leq v$ (in any other case $\mathbb{P}\left(\min _{s, t}=\min _{u, v}\right)=0$ ). In case (a), we have $\min _{s, t}=$ 
$\min _{u, v}=\min _{u, t}$ and so $\gamma_{s, t}=\gamma_{u, t}=\gamma_{u, v}$ by (11) and (12). Similarly in case (b), we have $\gamma_{s, t}=\gamma_{u, t}=\gamma_{u, v}$.

Proposition 5 Fix $s<t, s \in \mathbb{D}, n \geq 1$ and $\left\{\left(s_{i}, t_{i}\right) ; 1 \leq i \leq n\right\}$ with $s_{i}<t_{i}, s_{i} \in \mathbb{D}$. Then

(i) $\gamma_{s, t}$ is independent of $\sigma(W)$.

(ii) For all $i \in[1, N], h \in[1, n]$, we have

$$
E\left[1_{\left\{\gamma_{s, t}=\mathbf{e}_{i}\right\}} \mid\left(\gamma_{s_{i}, t_{i}}\right)_{1 \leq i \leq n}, W\right]=1_{\left\{\gamma_{s_{h}, t_{h}}=\mathbf{e}_{i}\right\}} \text { on }\left\{\min _{s, t}=\min _{s_{h}, t_{h}}\right\} .
$$

(iii) The law of $\gamma_{s, t}$ knowing $\left(\gamma_{s_{i}, t_{i}}\right)_{1 \leq i \leq n}$ and $W$ is given by $\sum_{i=1}^{N} \alpha_{i} \delta_{\mathbf{e}_{i}}$ when $\min _{s, t} \notin$

$$
\left\{\min _{s_{i}, t_{i}} ; 1 \leq i \leq n\right\} \text {. }
$$

This entirely describes the law of $\left(W, \psi_{s,}, s \in \mathbb{D}\right)$ in $\widetilde{C}$ independently of $\left(n_{k}, k \in \mathbb{N}\right)$ and consequently $Z^{n} \underset{n \rightarrow+\infty}{\stackrel{\text { law }}{\longrightarrow}} Z$ in $\widetilde{C}$.

Proof. (i) is clear. (ii) is a consequence of Proposition 4 (ii). (iii) Write $\left\{s, t, s_{i}, t_{i}, 1 \leq\right.$ $i \leq n\}=\left\{r_{k}, 1 \leq k \leq m\right\}$ with $r_{j}<r_{j+1}$ for all $1 \leq j \leq m-1$. Suppose that $s=$ $r_{i}, t=r_{h}$ with $i<h$. Then a.s. $\left\{\min _{r_{j}, r_{j+1}}, i \leq j \leq h-1\right\}$ are distinct and it will be sufficient to show that $\gamma_{s, t}$ is independent of $\sigma\left(\left(\gamma_{s_{i}, t_{i}}\right)_{1 \leq i \leq n}, W\right)$ conditionally to $A=\left\{\min _{s, t}=\min _{r_{j}, r_{j+1}}, \min _{s, t} \neq \min _{s_{i}, t_{i}}\right.$ for all $\left.1 \leq i \leq n\right\}$ for $j \in[i, h-1]$. On $A$, we have $\gamma_{s, t}=\gamma_{r_{j}, r_{j+1}},\left\{\min _{s_{i}, t_{i}}, 1 \leq i \leq n\right\} \subset\left\{\min _{r_{k}, r_{k+1}}, k \neq j\right\}$ and so $\left\{\gamma_{s_{i}, t_{i}}, 1 \leq\right.$ $i \leq n\} \subset\left\{\gamma_{r_{k}, r_{k+1}}, k \neq j\right\}$. Since $\gamma_{r_{1}, r_{2}}, \cdots, \gamma_{r_{m-1}, r_{m}}, W$ are independent, it is now easy to conclude.

In the sequel, we still assume that all processes are defined on the same probability space and that $Z^{n} \underset{n \rightarrow+\infty}{\stackrel{\text { a.s. }}{\longrightarrow}} Z$ in $\widetilde{C}$. In particular $\forall s \in \mathbb{D}, T>0$,

$$
\lim _{k \rightarrow+\infty} \sup _{s \leq t \leq s+T}\left|\frac{1}{\sqrt{k}} \Psi_{\lfloor k s\rfloor,\lfloor k t\rfloor}-\psi_{s, t}\right|=0 \text { a.s. }
$$

\subsubsection{Extension of the limit process.}

For a fixed $s<t, \min _{s, t}$ is attained in $] s, t[$ a.s. By Proposition 4 (ii), on a measurable set $\Omega_{s, t}$ with probability $1, \lim _{s^{\prime} \rightarrow s+, s^{\prime} \in \mathbb{D}} \gamma_{s^{\prime}, t}$ exists. Define $\varepsilon_{s, t}=\lim _{s^{\prime} \rightarrow s+, s^{\prime} \in \mathbb{D}} \gamma_{s^{\prime}, t}$ on $\Omega_{s, t}$ and give an arbitrary value to $\varepsilon_{s, t}$ on $\Omega_{s, t}^{c}$. Now, let $\varphi_{s, t}=\varepsilon_{s, t} W_{s, t}^{+}$. Then for all $s \in \mathbb{D}, t>s,\left(\varepsilon_{s, t}, \varphi_{s, t}\right)$ is a modification of $\left(\gamma_{s, t}, \psi_{s, t}\right)$. For all $s \in \mathbb{R}, t>s, \varphi_{s, t}=$ $\lim _{n \rightarrow \infty} \varphi_{s_{n}, t}$ a.s., where $s_{n}=\frac{\left\lfloor 2^{n} s\right\rfloor+1}{2^{n}}$ and therefore $\left(\varphi_{s, t}\right)_{t \geq s}$ is an $W\left(\alpha_{1}, \cdots, \alpha_{N}\right)$ started at 0 . Again, Proposition 4 (ii) yields

$$
\forall s<t, u<v, \mathbb{P}\left(\varepsilon_{s, t}=\varepsilon_{u, v} \mid \min _{s, t}=\min _{u, v}\right)=1 \text { if } \mathbb{P}\left(\min _{s, t}=\min _{u, v}\right)>0 .
$$

Define: 


$$
\varphi_{s, t}(x)=\left(x+\mathbf{e}(x) W_{s, t}\right) 1_{\left\{t \leq \tau_{s, x}\right\}}+\varphi_{s, t} 1_{\left\{t>\tau_{s, x}\right\}}, s \leq t, x \in G,
$$

where $W_{s, t}=W_{t}-W_{s}$ and $\tau_{s, x}$ is given by (4).

Proposition 6 Let $x \in G, x_{n} \in \frac{1}{\sqrt{n}} G_{\mathbb{N}}, \lim _{n \rightarrow \infty} x_{n}=x, s \in \mathbb{R}, T>0$. Then, we have

$$
\lim _{n \rightarrow+\infty} \sup _{s \leq t \leq s+T}\left|\frac{1}{\sqrt{n}} \Psi_{\lfloor n s\rfloor,\lfloor n t\rfloor}\left(\sqrt{n} x_{n}\right)-\varphi_{s, t}(x)\right|=0 \text { a.s. }
$$

Proof. Let $s^{\prime}$ be a dyadic number such that $s<s^{\prime}<s+T$. By (15), for $t>s^{\prime}$ :

$$
\left\{\min _{s, t}=\min _{s^{\prime}, t}\right\} \subset\left\{\varphi_{s, t}=\varphi_{s^{\prime}, t}\right\} \text { a.s. }
$$

and so, a.s.

$$
\forall t>s^{\prime}, t \in \mathbb{D} ;\left\{\min _{s, t}=\min _{s^{\prime}, t}\right\} \subset\left\{\varphi_{s, t}=\varphi_{s^{\prime}, t}\right\} .
$$

If $t>s^{\prime}, \min _{s, t}=\min _{s^{\prime}, t}$ and $t_{n} \in \mathbb{D}, t_{n} \downarrow t$ as $n \rightarrow \infty$, then $\min _{s, t_{n}}=\min _{s^{\prime}, t_{n}}$ which entails that $\varphi_{s, t_{n}}=\varphi_{s^{\prime}, t_{n}}$ and therefore $\varphi_{s, t}=\varphi_{s^{\prime}, t}$ by letting $n \rightarrow \infty$. This shows that a.s.

$$
\forall t>s^{\prime} ;\left\{\min _{s, t}=\min _{s^{\prime}, t}\right\} \subset\left\{\varphi_{s, t}=\varphi_{s^{\prime}, t}\right\} .
$$

As a result a.s.

$$
\left.\forall s^{\prime} \in \mathbb{D} \cap\right] s, s+T\left[, \forall t>s^{\prime} ;\left\{\min _{s, t}=\min _{s^{\prime}, t}\right\} \subset\left\{\varphi_{s, t}=\varphi_{s^{\prime}, t}\right\} .\right.
$$

By standard properties of Brownian paths, a.s. $\min _{s, s+T} \notin\left\{W_{s}, W_{s+T}\right\}$ and

$$
\left.\forall p \in \mathbb{N}^{*} ; \min _{s, s+\frac{1}{p}}<W_{s}, \min _{s, s+\frac{1}{p}} \neq W_{s+\frac{1}{p}}, \exists ! u_{p} \in\right] s, s+\frac{1}{p}\left[: \min _{s, s+\frac{1}{p}}=W_{u_{p}} .\right.
$$

The reasoning below holds almost surely: Take $p \geq 1, \min _{s, s+\frac{1}{p}}>\min _{s, s+T}$. Let $\left.\mathscr{S}_{p} \in\right] s, s+\frac{1}{p}\left[: \min _{s, s+\frac{1}{p}}=W_{\mathscr{S}_{p}}\right.$ and $s^{\prime}$ be a (random) dyadic number in $] s, \mathscr{S}_{p}[$. Then $\min _{s, s^{\prime}}>\min _{s^{\prime}, t}$ for all $t \in\left[\mathscr{S}_{p}, s+T\right]$. By uniform convergence:

$\exists n_{0} \in \mathbb{N}: \forall n \geq n_{0}, \forall \mathscr{S}_{p} \leq t \leq s+T, \min _{u \in\left[s, s^{\prime}\right]} S_{\lfloor n u\rfloor}>\min _{u \in\left[s^{\prime}, t\right]} S_{\lfloor n u\rfloor}$ and so $\Psi_{\left\lfloor n s^{\prime}\right\rfloor,\lfloor n t\rfloor}=\Psi_{\lfloor n s\rfloor,\lfloor n t\rfloor}$.

Therefore for $n \geq n_{0}$, we have

$$
\sup _{\mathscr{S}_{p} \leq t \leq s+T}\left|\frac{1}{\sqrt{n}} \Psi_{\lfloor n s\rfloor,\lfloor n t\rfloor}-\varphi_{s, t}\right|=\sup _{\mathscr{S}_{p} \leq t \leq s+T}\left|\frac{1}{\sqrt{n}} \Psi_{\left\lfloor n s^{\prime}\right\rfloor,\lfloor n t\rfloor}-\varphi_{s^{\prime}, t}\right| \text { (using (16)) }
$$

and so 


$$
\begin{aligned}
\sup _{s \leq t \leq s+T}\left|\frac{1}{\sqrt{n}} \Psi_{\lfloor n s\rfloor,\lfloor n t\rfloor}-\varphi_{s, t}\right| & \leq \sup _{s \leq t \leq \mathscr{S}_{p}}\left|\frac{1}{\sqrt{n}} \Psi_{\lfloor n s\rfloor,\lfloor n t\rfloor}-\varphi_{s, t}\right|+\sup _{\mathscr{S}_{p} \leq t \leq s+T}\left|\frac{1}{\sqrt{n}} \Psi_{\lfloor n s\rfloor,\lfloor n t\rfloor}-\varphi_{s, t}\right| \\
& \leq \sup _{s \leq t \leq s+\frac{1}{p}}\left(\frac{1}{\sqrt{n}} S_{\lfloor n s\rfloor,\lfloor n t\rfloor}^{+}+W_{s, t}^{+}\right)+\sup _{\mathscr{S}_{p} \leq t \leq s+T}\left|\frac{1}{\sqrt{n}} \Psi_{\left\lfloor n s^{\prime}\right\rfloor,\lfloor n t\rfloor}-\varphi_{s^{\prime}, t}\right| \\
& \leq \sup _{s \leq t \leq s+\frac{1}{p}}\left(\frac{1}{\sqrt{n}} S_{\lfloor n s\rfloor,\lfloor n t\rfloor}^{+}+W_{s, t}^{+}\right)+\sup _{s^{\prime} \leq t \leq s^{\prime}+T}\left|\frac{1}{\sqrt{n}} \Psi_{\left\lfloor n s^{\prime}\right\rfloor,\lfloor n t\rfloor}-\varphi_{s^{\prime}, t}\right| .
\end{aligned}
$$

From (14), a.s. $\forall u \in \mathbb{D}, \lim _{n \rightarrow+\infty} \sup _{u \leq t \leq u+T}\left|\frac{1}{\sqrt{n}} \Psi_{\lfloor n u\rfloor,\lfloor n t\rfloor}-\varphi_{u, t}\right|=0$. By letting $n$ go to $+\infty$ and then $p$ go to $+\infty$, we obtain

$$
\lim _{n \rightarrow \infty} \sup _{s \leq t \leq s+T}\left|\frac{1}{\sqrt{n}} \Psi_{\lfloor n s\rfloor,\lfloor n t\rfloor}-\varphi_{s, t}\right|=0 \text { a.s. }
$$

We now show that

$$
\lim _{n \rightarrow+\infty} \frac{1}{n} T_{\lfloor n s\rfloor, \sqrt{n} x_{n}}=\tau_{s, x} \text { a.s. }
$$

We have

For $\varepsilon>0$, from

$$
\frac{1}{n} T_{\lfloor n s\rfloor, \sqrt{n} x_{n}}=\inf \left\{r \geq \frac{\lfloor n s\rfloor}{n}: S_{r}^{n}-S_{s}^{n}=-\left|x_{n}\right|\right\} .
$$

$$
\lim _{n \rightarrow \infty} \sup _{u \in\left[\tau_{s, x}, \tau_{s, x}+\varepsilon\right]}\left|\left(S_{u}^{n}-S_{s}^{n}+\left|x_{n}\right|\right)-\left(W_{s, u}+|x|\right)\right|=0,
$$

we get

$$
\lim _{n \rightarrow \infty} \inf _{u \in\left[\tau_{s, x}, \tau_{s, x}+\varepsilon\right]}\left(S_{u}^{n}-S_{s}^{n}+\left|x_{n}\right|\right)=\inf _{u \in\left[\tau_{s, x}, \tau_{s, x}+\varepsilon\right]}\left(W_{s, u}+|x|\right)<0
$$

which implies $\frac{1}{n} T_{\lfloor n s\rfloor, \sqrt{n} x_{n}}<\tau_{s, x}+\varepsilon$ for $n$ large. If $x=0, \frac{1}{n} T_{\lfloor n s\rfloor, \sqrt{n} x_{n}} \geq \frac{\lfloor n s\rfloor}{n}$ entails obviously (18). If $x \neq 0$, then working in $\left[s, \tau_{s, x}-\varepsilon\right]$ as before and using $\inf _{u \in\left[s, \tau_{s, x}-\varepsilon\right]}\left(W_{u}-W_{s}+|x|\right)>0$, we prove that $\frac{1}{n} T_{\lfloor n s\rfloor, \sqrt{n} x_{n}} \leq \tau_{s, x}-\varepsilon$ for $n$ large which establishes (18).

Now

$$
\sup _{s \leq t \leq s+T}\left|\frac{1}{\sqrt{n}} \Psi_{\lfloor n s\rfloor,\lfloor n t\rfloor}\left(\sqrt{n} x_{n}\right)-\varphi_{s, t}(x)\right| \leq \sup _{s \leq t \leq s+T} Q_{s, t}^{1, n}+\sup _{s \leq t \leq s+T} Q_{s, t}^{2, n}
$$

where

$$
\begin{gathered}
Q_{s, t}^{1, n}=\mid\left(x_{n}+\mathbf{e}\left(x_{n}\right)\left(S_{t}^{n}-S_{s}^{n}\right)\right) 1_{\left\{\lfloor n t\rfloor \leq T_{\left.\lfloor n s\rfloor, \sqrt{n} x_{n}\right\}}-\left(x+\mathbf{e}(x) W_{s, t}\right) 1_{\left\{t \leq \tau_{s, x}\right\}} \mid,\right.} \\
Q_{s, t}^{2, n}=\mid \frac{1}{\sqrt{n}} \Psi_{\lfloor n s\rfloor,\lfloor n t\rfloor} 1_{\left\{\lfloor n t\rfloor>T_{\left.\lfloor n s\rfloor, \sqrt{n} x_{n}\right\}}-\varphi_{s, t} 1_{\left\{t>\tau_{s, x}\right\}} \mid .\right.}
\end{gathered}
$$


By (17), (18) and the convergence of $\frac{1}{\sqrt{n}} S_{\lfloor n .\rfloor}$ towards $W$ on compact sets, the righthand side of (19) converges to 0 when $n \rightarrow+\infty$.

Remark 2 From the definition of $\varepsilon_{s, t}$ (or Proposition 6), it is obvious that $\varepsilon_{r_{1}, r_{2}}, \cdots, \varepsilon_{r_{m-1}, r_{m}}, W$ are independent for all $r_{1}<\cdots<r_{m}$. Using (15), we easily check that (i), (ii) and (iii) of Proposition 5 are satisfied for all $s<t, n \geq$ $1,\left\{\left(s_{i}, t_{i}\right) ; 1 \leq i \leq n\right\}$ with $s_{i}<t_{i}$ (the proof remains the same as Proposition 5).

Proposition $7 \varphi$ is the unique stochastic flow of mappings solution of $(T)$.

Proof. Fix $s<t<u, x \in G$ and let prove that $\varphi_{s, u}(x)=\varphi_{t, u} \circ \varphi_{s, t}(x)$ a.s. We follow Lemma 4.3 [7] and denote $\tau_{s, x}$ by $\tau_{s}(x)$. All the equalities below hold a.s.

On the event $\left\{u<\tau_{s}(x)\right\}, \varphi_{s, t}(x)=x+\mathbf{e}(x) W_{s, t}, \tau_{t}\left(\varphi_{s, t}(x)\right)=\tau_{s}(x)<u$ and

$$
\varphi_{t, u} \circ \varphi_{s, t}(x)=x+\mathbf{e}(x)\left(W_{s, t}+W_{t, u}\right)=x+\mathbf{e}(x) W_{s, u}=\varphi_{s, u}(x) .
$$

On the event $\left.\left.\left\{\tau_{s}(x) \in\right] t, u\right]\right\}$, we still have $\varphi_{s, t}(x)=x+\mathbf{e}(x) W_{s, t}$ and $\tau_{t}\left(\varphi_{s, t}(x)\right)=$ $\tau_{s}(x) \leq u$, thus

$$
\varphi_{t, u} \circ \varphi_{s, t}(x)=\varepsilon_{t, u} W_{t, u}^{+}=\varepsilon_{s, u} W_{s, u}^{+}=\varphi_{s, u}(x) .
$$

since on the event $\left.\left.\left\{\tau_{s}(x) \in\right] t, u\right]\right\}, \min _{s, u}=\min _{t, u}$ and $W_{s, u}^{+}=W_{u}-\min _{s, u}=W_{t, u}^{+}$.

On the event $\left\{\tau_{s}(x) \leq t\right\} \cap\left\{\tau_{t}\left(\varphi_{s, t}(x)\right) \leq u\right\}, \varphi_{s, t}(x)=\varepsilon_{s, t} W_{s, t}^{+}$and

$$
\varphi_{t, u} \circ \varphi_{s, t}(x)=\varphi_{t, u}\left(\varepsilon_{s, t} W_{s, t}^{+}\right)=\varepsilon_{t, u} W_{t, u}^{+}=\varepsilon_{s, u} W_{s, u}^{+}=\varphi_{s, u}(x)
$$

since $W_{s, \tau_{t}\left(\varphi_{s, t}(x)\right)}^{+}=0$ and thus $\min _{s, u}=\min _{t, u}$ which implies $\varepsilon_{s, u}=\varepsilon_{t, u}$ and $W_{s, u}^{+}=$ $W_{t, u}^{+}$.

On the event $\left\{\tau_{s}(x) \leq t\right\} \cap\left\{\tau_{t}\left(\varphi_{s, t}(x)\right)>u\right\}, \varphi_{s, t}(x)=\varepsilon_{s, t} W_{s, t}^{+}$and

$$
\varphi_{t, u} \circ \varphi_{s, t}(x)=\varphi_{t, u}\left(\varepsilon_{s, t} W_{s, t}^{+}\right)=\varepsilon_{s, t}\left(W_{s, t}^{+}+W_{t, u}\right)=\varepsilon_{s, u} W_{s, u}^{+}=\varphi_{s, u}(x) .
$$

since in this case $\min _{s, u}=\min _{s, t}$ which implies $\varepsilon_{s, u}=\varepsilon_{s, t}$ and

$$
\begin{aligned}
W_{s, u}^{+} & =W_{u}-\min _{s, u} \\
& =W_{u}-W_{s}+W_{s}-\min _{s, t} \\
& =W_{s, t}^{+}+W_{t, u}
\end{aligned}
$$

Thus we have, a.s. $\varphi_{s, u}(x)=\varphi_{t, u} \circ \varphi_{s, t}(x)$ which proves the cocyle property for $\varphi$. It is now easy to check that $\varphi$ is a stochastic flow of mappings in the sense of Definition $4[5]$.

Note that $\left(\varphi_{0, t}, t \geq 0\right)$ is an $W\left(\alpha_{1}, \cdots, \alpha_{N}\right)$ started at 0 and therefore satisfies Freidlin-Sheu formula (Theorem 3 [5]). Let $f \in D\left(\alpha_{1}, \cdots, \alpha_{N}\right)$, then for all $t \geq 0$,

$$
f\left(\varphi_{0, t}\right)=f(0)+\int_{0}^{t} f^{\prime}\left(\varphi_{0, u}\right) d B_{u}+\frac{1}{2} \int_{0}^{t} f^{\prime \prime}\left(\varphi_{0, u}\right) d u \text { a.s. }
$$


where $B_{t}=\left|\varphi_{0, t}\right|-\tilde{L}_{t}\left(\left|\varphi_{0,}\right|\right)$ and $\tilde{L}_{t}\left(\left|\varphi_{0, .}\right|\right)$ is the symmetric local time at 0 of $\left|\varphi_{0, .}\right|$. Since $\left|\varphi_{0, t}\right|=W_{t}-\min _{0, t}$, we get $B_{t}=W_{t}$. Let $x \in D_{i} \backslash\{0\}$ and $f_{i}(r)=f\left(r \mathbf{e}_{i}\right), r \geq 0$. Since $\lim _{z \rightarrow 0, z \in D_{i}, z \neq 0} f^{\prime}(z)$ and $\lim _{z \rightarrow 0, z \in D_{i}, z \neq 0} f^{\prime \prime}(z)$ exist, we can construct $g$ which is $C^{2}$ on $\mathbb{R}$ and coincides with $f_{i}$ on $\mathbb{R}_{+}$. By Itô's formula

$$
g\left(|x|+W_{t}\right)=g(|x|)+\int_{0}^{t} g^{\prime}\left(|x|+W_{u}\right) d W_{u}+\frac{1}{2} \int_{0}^{t} g^{\prime \prime}\left(|x|+W_{u}\right) d u \text { a.s. }
$$

and so for $t \leqslant \tau_{0}(x)$, we have

$$
f\left(\varphi_{0, t}(x)\right)=f(x)+\int_{0}^{t} f^{\prime}\left(\varphi_{0, u}(x)\right) d W_{u}+\frac{1}{2} \int_{0}^{t} f^{\prime \prime}\left(\varphi_{0, u}(x)\right) d u \text { a.s. }
$$

Set $\alpha=f(0)+\int_{0}^{\tau_{0}(x)} f^{\prime}\left(\varphi_{0, u}\right) d W_{u}+\frac{1}{2} \int_{0}^{\tau_{0}(x)} f^{\prime \prime}\left(\varphi_{0, u}\right) d u=f\left(\varphi_{0, \tau_{0}(x)}\right)=f(0)$ since $W_{0, \tau_{0}(x)}^{+}=0$. Then for $t>\tau_{0}(x)$, write

$$
\begin{aligned}
f\left(\varphi_{0, t}(x)\right)=f\left(\varphi_{0, t}\right) & =\alpha+\int_{\tau_{0}(x)}^{t} f^{\prime}\left(\varphi_{0, u}\right) d W_{u}+\frac{1}{2} \int_{\tau_{0}(x)}^{t} f^{\prime \prime}\left(\varphi_{0, u}\right) d u \\
& =f(0)+\int_{\tau_{0}(x)}^{t} f^{\prime}\left(\varphi_{0, u}(x)\right) d W_{u}+\frac{1}{2} \int_{\tau_{0}(x)}^{t} f^{\prime \prime}\left(\varphi_{0, u}(x)\right) d u .
\end{aligned}
$$

But $f(x)+\int_{0}^{\tau_{0}(x)} f^{\prime}\left(\varphi_{0, u}(x)\right) d W_{u}+\frac{1}{2} \int_{0}^{\tau_{0}(x)} f^{\prime \prime}\left(\varphi_{0, u}(x)\right) d u=f\left(\varphi_{0, \tau_{0}(x)}(x)\right)=f(0)$ and so, for all $t \geq 0, f \in D\left(\alpha_{1}, \cdots, \alpha_{N}\right), x \in G$,

$$
f\left(\varphi_{0, t}(x)\right)=f(x)+\int_{0}^{t} f^{\prime}\left(\varphi_{0, u}(x)\right) d W_{u}+\frac{1}{2} \int_{0}^{t} f^{\prime \prime}\left(\varphi_{0, u}(x)\right) d u \text { a.s. }
$$

Now, let $(\psi, W)$ be a any flow of mappings solution of $(T)$. Lemma 6 [5] implies

$$
\psi_{0, t}(x)=x+\mathbf{e}(x) W_{0, t} \text { for } 0 \leq t \leq \tau_{0, x} \text { with } \tau_{0, x} \text { given by (4). }
$$

By considering a sequence $\left(x_{k}\right)_{k \geq 0}$ converging to $\infty$, this shows that $\sigma\left(W_{t}\right) \subset$ $\sigma\left(\psi_{0, t}(y), y \in G\right)$. Therefore, we can define a Wiener stochastic flow $K^{*}$ obtained by filtering $\delta_{\psi}$ with respect to $\sigma(W)$ (Lemma 3-2 (ii) in [6]) satisfying: $\forall s \leq t, x \in$ $G, K_{s, t}^{*}(x)=E\left[\delta_{\psi_{s, t}(x)} \mid \sigma(W)\right]$ a.s. In particular $K^{*}$ solves $(T)$ and since $K^{W}$ given by (3) is the unique Wiener solution of $(T)$, we get: $\forall s \leq t, x \in G, \quad K_{s, t}^{W}(x)=$ $E\left[\delta_{\psi_{s, t}(x)} \mid \sigma(W)\right]$ a.s. (see Proposition 8 [5]). As $K_{0, t}^{W}(0)$ is supported on $\left\{W_{0, t}^{+} \mathbf{e}_{i}, \quad 1 \leq\right.$ $i \leq N\}$, we deduce that $\left|\psi_{0, t}(0)\right|=W_{0, t}^{+}$. Combining this with (21), we see that

$$
\inf \left\{r \geq 0: \psi_{0, r}(x)=\psi_{0, r}(0)\right\}=\tau_{0, x} .
$$

This implies $\psi_{0, r}(x)=\psi_{0, r}(0)$ for all $r \geq \tau_{0, x}$ by applying the following

Lemma 2. For all $\left(x_{1}, \cdots, x_{n}\right) \in G^{n}$, denote by $\mathbb{P}_{x_{1}, \cdots, x_{n}}$ the law of $\left(\psi_{0, .}\left(x_{1}\right), \cdots, \psi_{0, .}\left(x_{n}\right)\right)$ in $C\left(\mathbb{R}_{+}, G^{n}\right)$. Let $T$ be a finite $\left(\mathscr{F}_{t}\right)$ stopping time where $\mathscr{F}_{t}=\sigma\left(\psi_{0, u}, u \leq\right.$ $t), t \geq 0$. Then the law of $\left(\psi_{0, T+} .\left(x_{1}\right), \cdots, \psi_{0, T+} .\left(x_{n}\right)\right)$ knowing $\mathscr{F}_{T}$ is given by $\mathbb{P}_{\psi_{0, T}\left(x_{1}\right), \cdots, \psi_{0, T}\left(x_{n}\right)}$. 


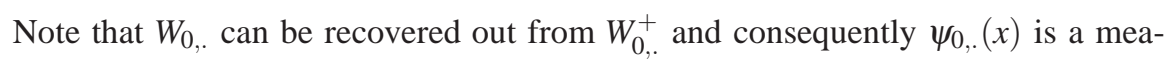
surable function of $\psi_{0, .}(0)$ for all $x \in G$. Therefore, for all $\left(x_{1}, \cdots, x_{n}\right) \in G^{n}$, $\left(\psi_{0, \cdot}\left(x_{1}\right), \cdots, \psi_{0}, \cdot\left(x_{n}\right)\right)$ is unique in law since $\psi_{0, \cdot}(0)$ is a Walsh Brownian motion. This completes the proof.

\subsubsection{The Wiener flow.}

Remark that $K_{s, t}^{W}(x)=E\left[\delta_{\varphi_{s, t}(x)} \mid \sigma(W)\right]$ which entails that $K^{W}$ is a stochastic flow of kernels. By conditioning with respect to $\sigma(W)$ in $(20)$, we easily see that $\left(K^{W}, W\right)$ solves $(T)$. In order to finish the proof of Theorem 2 and Corollary 1 , we need only check the following lemma (the proof of (6) is similar)

Lemma 3. Under the hypothesis of Proposition 6, we have

$$
\sup _{t \in[s, s+T]} \beta\left(K_{s, t}^{W}(x), K_{s, t}^{n}\left(\sqrt{n} x_{n}\right)\right) \underset{n \rightarrow+\infty}{\longrightarrow} 0 \text { a.s. }
$$

Proof. Let $g: G \longrightarrow \mathbb{R}$ such that $\|g\|_{\infty}+\sup _{x \neq y} \frac{|g(x)-g(y)|}{|x-y|} \leq 1, g(0)=0$. Then,

$$
\left|\int_{G} g(y) K_{s, t}^{W}(x)(d y)-\int_{G} g(y) K_{s, t}^{n}\left(\sqrt{n} x_{n}\right)(d y)\right| \leq V_{s, t}^{1, n}+V_{s, t}^{2, n}
$$

where

$$
\begin{gathered}
V_{s, t}^{1, n}=\left|g\left(x_{n}+\mathbf{e}\left(x_{n}\right) S_{s, t}^{n}\right) 1_{\left\{\lfloor n t\rfloor \leq T_{\lfloor n s\rfloor, \sqrt{n} x_{n}}\right\}}-g\left(x+\mathbf{e}(x) W_{s, t}\right) 1_{\left\{t \leq \tau_{s, x}\right\}}\right|, \\
V_{s, t}^{2, n}=\sum_{j=1}^{N} \alpha_{j}\left|g\left(\mathbf{e}_{j} W_{s, t}^{+}\right) 1_{\left\{t>\tau_{s, x}\right\}}-g\left(\mathbf{e}_{j} S_{n, s, t}^{+}+o_{n}\right) 1_{\left\{\lfloor n t\rfloor>T_{\lfloor n s\rfloor, \sqrt{n} x_{n}}\right\}}\right|
\end{gathered}
$$

and $o_{n} \in G$ is a $\sigma(S)$ measurable random variable such that $\left|o_{n}\right| \leq \frac{1}{\sqrt{n}}, S_{s, t}^{n}=S_{t}^{n}-$ $S_{s}^{n}, S_{n, s, t}^{+}=\frac{1}{\sqrt{n}} S_{\lfloor n s\rfloor,\lfloor n t\rfloor}^{+}$. As $\lfloor x\rfloor-1 \leq x \leq\lfloor x\rfloor+1$ for all $x \in \mathbb{R}$, we get

$$
\begin{aligned}
& V_{s, t}^{1, n} \leq \sup _{t \in I_{n, s, x}}\left|x_{n}+\mathbf{e}\left(x_{n}\right) S_{s, t}^{(n)}-x-\mathbf{e}(x) W_{s, t}\right|+\sup _{t \in J_{n, s, x}}\left|g\left(x_{n}+\mathbf{e}\left(x_{n}\right) S_{s, t}^{(n)}\right)\right|+\sup _{t \in K_{n, s, x}}\left|g\left(x+\mathbf{e}(x) W_{s, t}\right)\right| \\
& \text { with } \\
& \qquad I_{n, s, x}=\left[s, \tau_{s, x} \vee\left(\frac{1}{n}+\frac{1}{n} T_{\lfloor n s\rfloor, \sqrt{n} x_{n}}\right)\right], \\
& \qquad J_{n, s, x}=\left[\tau_{s, x},\left(\frac{1}{n} T_{\lfloor n s\rfloor, \sqrt{n} x_{n}}+\frac{1}{n}\right) \vee \tau_{s, x}\right], \quad K_{n, s, x}=\left[\tau_{s, x} \wedge\left(\frac{1}{n} T_{\lfloor n s\rfloor, \sqrt{n} x_{n}}-\frac{1}{n}\right), \tau_{s, x}\right] .
\end{aligned}
$$

Using $|g(y)| \leq|y|$, we obtain

$$
\sup _{t \in J_{n, s, x}}\left|g\left(x_{n}+\mathbf{e}\left(x_{n}\right) S_{s, t}^{(n)}\right)\right|+\sup _{t \in K_{n, s, x}}\left|g\left(x+\mathbf{e}(x) W_{s, t}\right)\right| \leq \sup _{t \in J_{n, s, x}}|| x_{n}\left|+S_{s, t}^{(n)}\right|+\sup _{t \in K_{n, s, x}}|| x\left|+W_{s, t}\right| .
$$


Since $\lim _{n \rightarrow+\infty} \frac{1}{n} T_{\lfloor n s\rfloor, \sqrt{n} x_{n}}=\tau_{s, x}$ a.s., the right-hand side converges to 0 . By discussing the cases $x=0, x \neq 0$, we easily see that $\lim _{n \rightarrow \infty} \sup _{t \in I_{n, s, x}} \mid x_{n}+\mathbf{e}\left(x_{n}\right) S_{s, t}^{(n)}-x-$ $\mathbf{e}(x) W_{s, t} \mid=0$ and therefore $\lim _{n \rightarrow \infty} \sup _{t \in[s, s+T]} V_{s, t}^{1, n}=0$. By the same manner, we arrive at $\lim _{n \rightarrow \infty} \sup _{t \in[s, s+T]} V_{s, t}^{2, n}=0$ which proves the lemma.

Acknowledgements I sincerely thank Yves Le Jan, Olivier Raimond and Sophie Lemaire for very useful discussions. I am also grateful to the referee for his helpful comments.

\section{References}

1. Cherny, A. S. and Shiryaev, A. N. and Yor, M.: Limit behaviour of the "horizontal-vertical" random walk and some extensions of the Donsker-Prokhorov invariance principle. Teor. Veroyatnost. i Primenen 47(3), 498-517 (2002).

2. Enriquez, N. and Kifer, Y.: Markov chains on graphs and Brownian motion. J. Theoret. Probab. 14 (2), 495-510 (2001).

3. Ethier, S. and Kurtz, T.: Markov processes: Characterization and convergence. Wiley Series in Probability and Mathematical Statistics (1986).

4. Harrison, J. M. and Shepp, L. A.: On skew Brownian motion. Ann. Probab 9 (2) 309-313 (1981)

5. Hajri H.: Stochastic flows related to Walsh Brownian motion. Electronic journal of probability 16, 1563-1599 (2011).

6. Le Jan, Y. and Raimond, O.: Flows, coalescence and noise. Ann. Probab. 32 (2), 1247-1315, 2004.

7. Le Jan, Y. and Raimond, O.: Flows associated to Tanaka's SDE. ALEA Lat. Am. J. Probab. Math. Stat. (1), 21-34 (2006).

8. Nishchenko, I.I.: Discrete time approximation of coalescing stochastic flows on the real line. Theory of stochastic processes. 2011, 70-78.

9. Révész, P.: Random walk in random and non-random environments. Second edition (2005).

10. Revuz, D. and Yor, M.: Continuous martingales and Brownian motion, Grundlehren der Mathematischen Wissenschaften. Third edition (1999).

11. Watanabe, S.: The stochastic flow and the noise associated to Tanaka's stochastic differential equation. Ukraïn. Mat. Zh. 52 (9). 1176-1193 (2000). 